\title{
"The Oxidative Reaction of Potassium Permanganate with Mycolic Acids Leads to a Unique Diagnostic Pattern for Mycobacterium Tuberculosis"
}

\author{
"By Seyed Mohsen Khatami"
}

\begin{abstract}
In an oxidative reaction, potassium permanganate reacts with mycolic acid component of $M$.tuberculosis and gives rise to a unique saponified pattern specific for M.tuberculosis. This brown orange pattern make possible to identify M.tuberculosis in a typical laboratory sample. The method avoids the complexity of other methods with special regard to save time and costs.
\end{abstract}

\section{Introduction}

Mycobacterium tuberculosis, the causative agent of tuberculosis (TB), killed approximately two million people worldwide in 1997 and has infected approximately $32 \%$ of the world's population (Dye et al., 1999). The unique characteristics of $\mathrm{M}$. tuberculosis with special regards to its pathogenic consequences depend on unique characteristics of cell wall skeleton.

The isolated cell wall contains three heteropolymeric subunits composed of peptidoglycan, arabinogalactan (a complex heteropolymer composed primarily of arabinose and galactose), and mycolic acids. The currently accepted model of cell wall structure relegates the peptidoglycan and arabinosgalactan polymers largely to providing a scaffold for attaching and orienting mycolic acids in a direction perpendicular to the plane of the cell surface $^{1}$ (see Fig 2).

Other than the normal barrier functions of a cell wall skeleton involved in maintaining a rigid cell shape and turgor pressure against the variable osmolarity of the surrounding environment, mycobacteria and related genera possess many unique characteristics which are directly attributed to the presence of mycolic acids in their cell wall.2, 3 The more important characteristics conferred by this structure include properties such as resistance to chemical injury, low permeability to hydrophobic antibiotic substances, 4 resistance to dehydration, and the ability to persist and thrive within the hostile environment of the macrophage phaglysosome.5-7

Mycolic acids are very long chain $\alpha$-alkyl, $\beta$-hydroxy fatty acids that are unique to mycobacteria and are greater than 80 carbons in $\mathrm{Mtb}^{8}$. Mycolic acid melts at $53-55 \cdot 5^{\circ}$, has a molecular weight of 1316 and its formula indicates $\mathrm{C}_{88} \mathrm{H}_{172} \mathrm{O}_{4}$ or $\mathrm{C}_{88} \mathrm{H}_{176} \mathrm{O}_{4}$. 
The mycolate acids of the Mycobacteria are among the longest and most highly functionalized of the mycolata. They occur with a wide variety of functional groups, both polar and non-polar, which give rise to the characteristics HPLC profiles. These modifications occur at two points in the mero chain, referred to as distal (closest to the $\omega$-end of the chain) and proximal (closest to the $\beta$-hydroxy acid) ${ }^{1}$.

The most widespread of the classes of mycolic acids are the a mycolates, which may contain double bonds or cyclopropanes in cis configurations or double bonds or cyclopropanes in trans configurations with an adjacent methyl branch. Every mycobacterial species which has been examined to date produces a mycolates. The next most widely distributed class of mycolic acids are the ketomycolates. These are found in many different species; regardless of growth rate or status as pathogens or saprophytes.Methoxymycolates appear, with five exceptions, to be present only in pathogenic species, and primarily in slow-growers. Interestingly, methoxymycolates in all cases appear to co-occur in species which also produce ketomycloate ${ }^{1}$.

Oxygenated mycolates such as ketomycolate and methoxymycolate are extremely widespread and only a handful of species appear to be capable of maintaining cell wall function without the presence of such mycolates.

In spite of this fact there is very little actual evidence to suggest what physiological role such modifications play in the cell wall.

Ketomycolate is likely to be very active as a hydrogen-bond acceptor. Methoxymycolate, which has only an ether linkage, is less likely to be very active in this regard. It is noteworthy that the combination of a mycolate with methoxymycolate as the only oxygenated species is not a naturally-occurring combination, whereas the combination of only $\alpha$ and ketomycolate does occur $^{1}$.

M. tuberculosis produces three main types of mycolic acids: alpha-, methoxy-, and keto-. Alpha-mycolic acids comprise at least 70\% of the mycolic acids present in the organism and contain several cyclopropane rings. Methoxymycolic acids, which contain several methoxy groups, comprise between $10 \%$ and $15 \%$ of the mycolic acids in the organism. The remaining $10 \%$ to $15 \%$ of the mycolic acids are keto-mycolic acids, which contain several ketone groups. The presence of mycolic acids gives $M$. tuberculosis many characteristics that defy medical treatment. They lend the organism increased resistance to chemical damage and dehydration, and prevent the effective activity of hydrophobic antibiotics (see Fig 2). In addition, the mycolic acids allow the bacterium to grow readily inside macrophages, effectively hiding it from the host's immune system ${ }^{1,9}$. In pathogenic mycobacteria, but not in nonpathogenic species, mycolic acids are modified with cyclopropyl groups at relatively conserved positions in the meromycolate chain (see Fig 1).

These cyclopropane residues are added to Mtb mycolic acids by a family of SAdenosy Methionine dependent methyltransferases that exhibit exquisite substrate specificity for their lipid substrates ${ }^{10,11}$. Although cyclopropanation of mycolic acids is observed only in pathogenic mycobacterium such as $\mathrm{Tb}$ complex, little is known about the physiological function of this modification.

Potassium permanganate is highly reactive under normal chemical coundition.It will oxidize a wide variety of inorganic and organic substances. 
Potassium permanganate $(\mathrm{Mn} 7+)$ is reduced to mangances dioxide $(\mathrm{MnO} 2)$ (Mn 4+) which precipitates out of solution (Hazen and Sawer, 1992). All reactions are exothermic.

Under normal or alkaline condition, the half-reaction is (CRC, 1990):

$$
\mathrm{MnO}_{4}{ }^{i}+2 \mathrm{H}_{2} \mathrm{O}+3 e^{-} \rightarrow \mathrm{MnO}_{2}+4 \mathrm{OH}
$$

$E^{\circ}=0.60 \mathrm{~V}$

Potassium permanganate oxidizes lipids at the site of double bonds and therefore can be used to determine the position of unsaturations in lipid chains. According ALEX LESUKt AND R. J. ANDERSON "It is evident that oxidation of mycolic acid by permanganate was not sufficiently drastic to break down the large mycolic acid molecule into smaller recognizable units" but they emphasize "the principal product that was recovered differed but slightly in melting point and equivalent weight from the original acid. A small quantity of acids which had an equivalent weight of 741 and melted at 61- 68” was isolated but the material was non-crystalline and was apparently a mixture $12 "$. The oxidizing function of potassium permanganate leads to its reduction, creating an alkaline condition proper enough for creation of a saponification of fatty acid, lipid and ester. Saponification is the hydrolysis of an ester under basic conditions to form an alcohol and the salt of a carboxylic acid. Saponification is commonly used to refer to the reaction of a metallic alkali (base) with a fat or oil to form soap. Saponifiable substances are those that can be converted into soap. The oxidation of mycolic acids by potassium permanganate create such condition that lead to occurrence of a saponification process. we, in this experiment will use this characteristic as a prefect method in order to easily, rapidly and cheaply identify M.tuberculosis in clinical samples compared with other methods.

\section{Material and methods}

We used in our experiment of very strongly positive sample confirmed by PCR, culture and smear versus a negative sample confirmed by PCR, culture and smear. The used potassium permanganate was of high concentration. In the following we explain the procedures we developed for the objective of the experiment.

1-One milliliter of both positive and negative samples of type bronchial specimen were centrifuged at $12000 \mathrm{rpm}$ for 5 minutes. 2-The supernatants were discarded.

3 -The pellets were suspended in 500 micro liter mucolysin and processed by shaking under 5 minutes at room temperture.

4-The contents of tubes were centrifuged again at 12000 rpm for 5 minutes. 5 -The supernatants were discarded and the pellets were washed two times by 500 micro liter sterile water as the above-mentioned procedures in order to washing away the remaining of mucolysin and finally the pellets suspended in $\max \quad 15$ micro liter sterile water.

6-10 micro liters of suspensions were placed on two opposite and adjacent points of a microscopic slide and incubated at $56^{\circ} \mathrm{C}$ under 6 minutes in order 
to fix the mycobacterium tuberculosis in positive sample on microscopic slide.

7-When the slide was dehydrated, it was transferred to distillated water for two seconds and the slide again dried at room temperture.

8-When the slide was dehydrated at room temperature, we transferred 5 micro liters sterile water on both dehydrated points and added 10 micro liters highly concentrated potassium permanganate under mixing condition. The slide incubated again at $56^{\circ} \mathrm{C}$ until the slide was dehydrated.

9-The dehydrated points on slide washed by sterile water until the excess of color of potassium permanganate removed.

10-After drying the slide at room temperature the slide subjected for microscopic investigation.

\section{Result:}

The microscopic investigation provided a distinguishing difference between the positive sample and negative sample. The positive sample characterized by lightly deep to deep brown pattern indicating the consequences of oxidation of mycolic acids from M.tuberculosis by potassium permanganate described above. Such pattern was not observed in negative sample (Fig 3).

\section{Discussion}

The results of our experiment indicate that the oxidation of mycolic acids from M.tuberculosis by potassium permanganate inspire a perspective for emergence of a relatively easy, time-saving and cheap method intending the identification of M.tuberculosis compared with other methods including PCR and Ziehl-Neelsen staining and etc . A statistically raw analysis of more than hundred samples indicates that the specificity of this method is less than $50 \%$ compared with the golden standard but the sensitivity of this method is very high. The logic interpretation of this analysis is that not necessarily all positive indicated samples by this method can be confirmed by culture, PCR or other method but all negative indicated samples can be confirmed by culture, PCR and other methods. . We are statistically engaged to determine the magnitude of accuracy the method can provide.

I express my gratitude to the head of Baqiyatallah Clinical Laboratory and other kind colleagues for the fine cooperation during the performance of this work. Relating every question in connection with this work can correspondence be done through the e-mail address: mohsen_khatami3@yahoo.com

\section{References}

1. Barry et al. (1998). "Mycolic acids: structure, biosynthesis, and physiological functions." Prog. Lipid Res. 37(3): 143-79.

2. Minnikin, D. E., in The Biology of the Mycobacteria, Vol, eds C. Ratledge and J. Standford. Academic Press, London, 1982, pp. 95-184.

3. Rastogi, N., Res. Microbial., 1991, 142, 464-476. 
4. Nikaido, H., Science, 1994, 264, 382-388.

5. Barry, C. E., III and Mdluli., Trends Microbiol., 1996, 4, 275-281.

6. Clemens, D. L., Trends Microbiol., 1997, 5, 383-385.

7. Armstrong, J. A. and D'Arcy Hart, P., J. Exp. Med., 1971, 134, 713-740

8. Barry, C. E., 3rd, Lee, R. E., Mdluli, K., Sampson, A. E., Schroeder, B. G.,

9. Takayama, Wang, and Besra (2005). "Pathway to synthesis and processing of mycolic acids in Mycobacterium tuberculosis." Clin. Microbiol. Rev. 18(1): 81-101.

10. Barry, C. E., 3rd, Lee, R. E., Mdluli, K., Sampson, A. E., Schroeder, B. G., 11. Schroeder, B. G., and Barry, C. E., 3rd. (2001) Bioorg Chem 29, 164-177

12. ALEX LESUKt AND R. J. ANDERSON 'THE CHEMISTRY OF THE LIPIDS OF TUBERCLE BACILLI'(From the Department of Chemistry, Yale University, New Haven), (Received for publication, July 26, 1940 in the journal of biological chemistry).

\section{Figure legends}

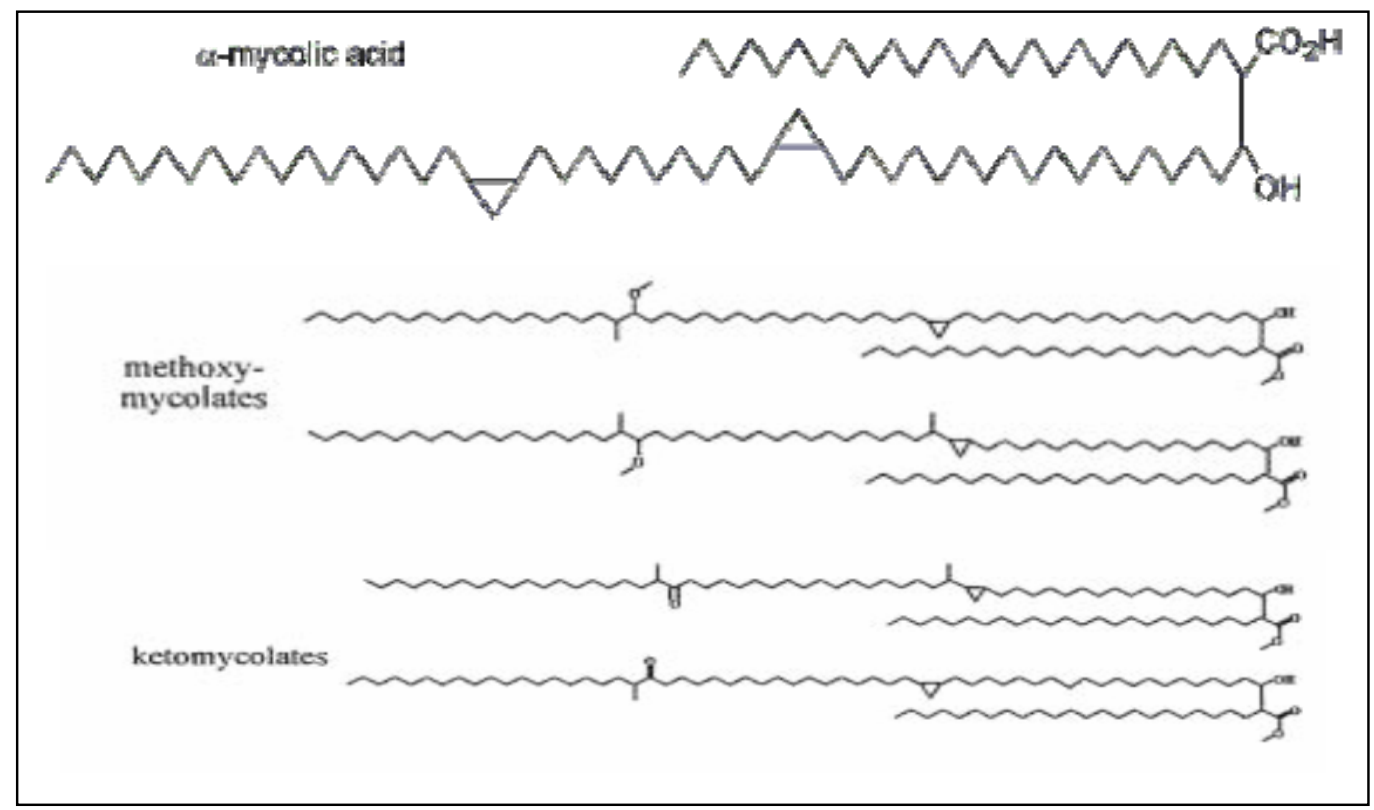

Figure 1: An example of a A) $\alpha$-mycolic acid, B) Methoxy mycolates, C) Ketomycolates from Mycobacterium tuberculosis 


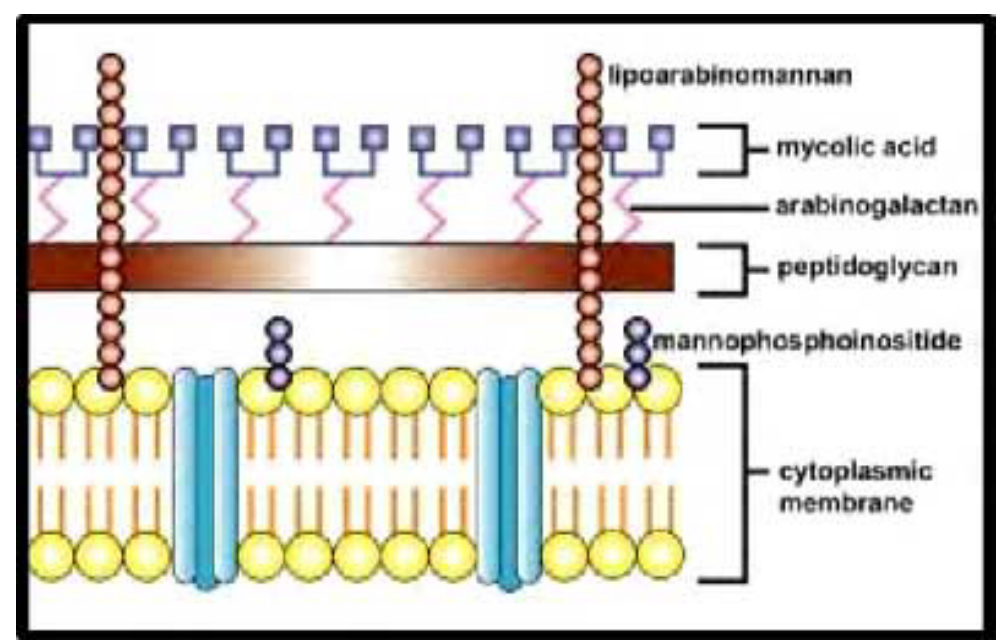

Figure 2: Schematic diagram of the cell envelope of M.tuberculosis.Exteroir to the cell membrane (CM) and peptidoglycan (PG), mycolic acids are covalently linked to the arabinogalactan (AG), forming a thick hydrophobic layer. Mycolic acids in the form of trehalose dimycolate (TDM) are noncovalently associated with the cell envelope. 


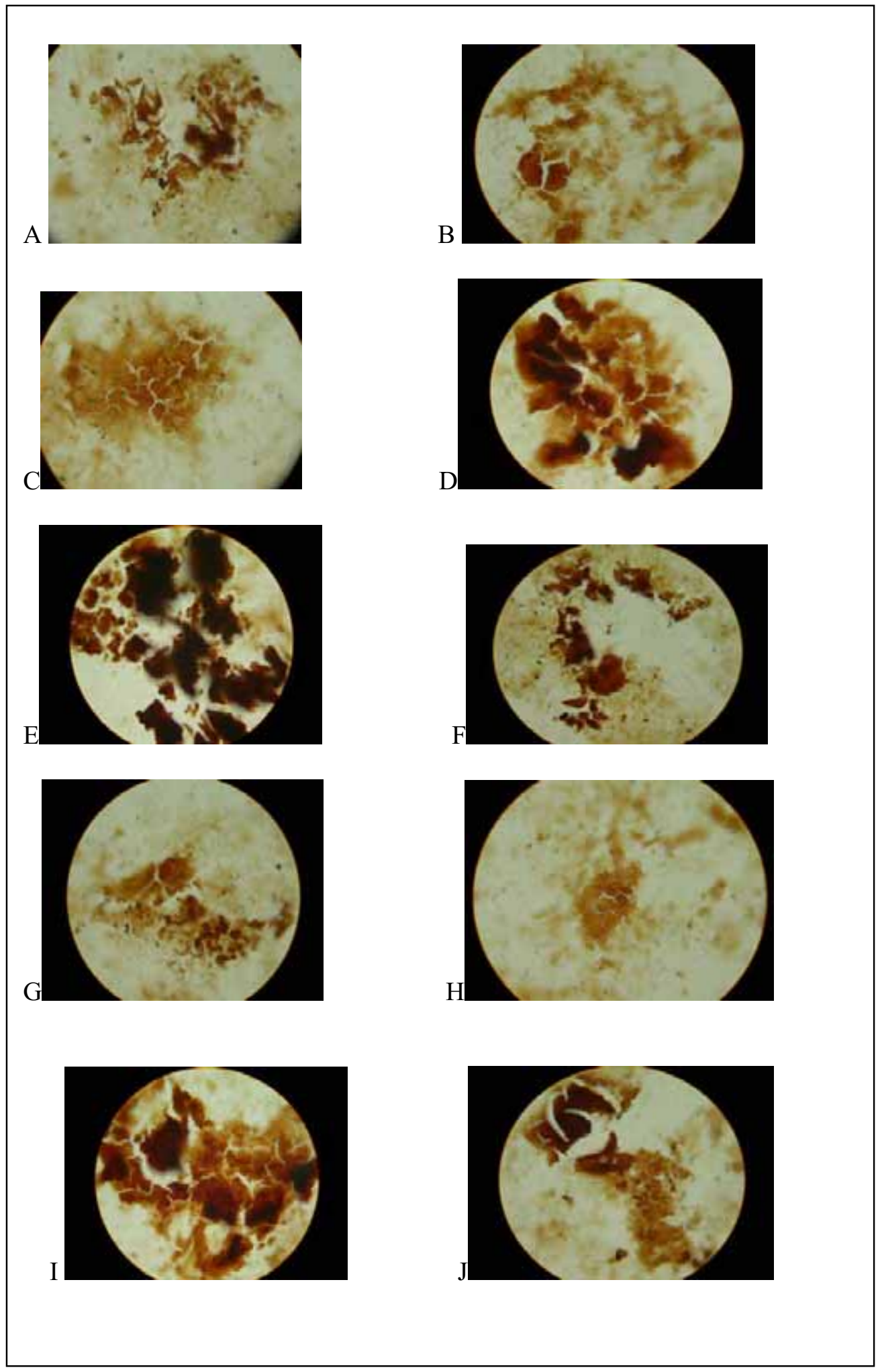

Figure 3: A, B, C, D, E, F, G, H, I and J indicating positive samples. 


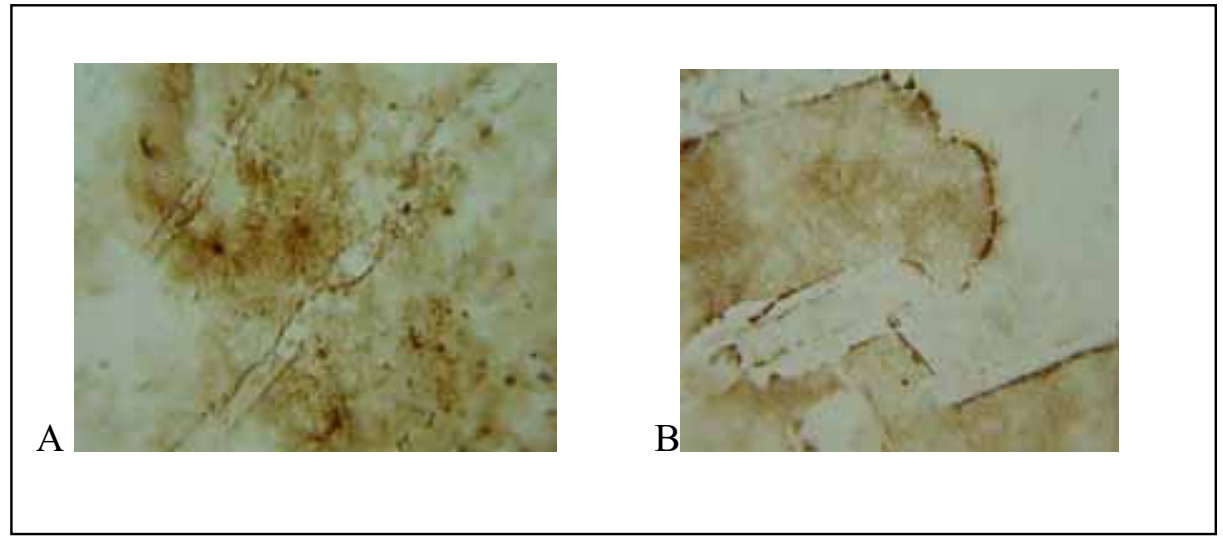

Figure 4: A and B indicating negative samples. 